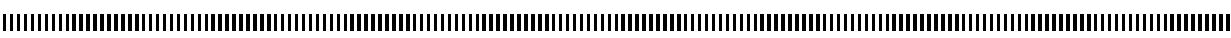

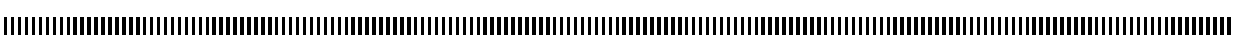
|

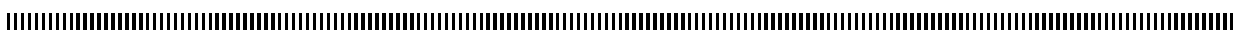
|

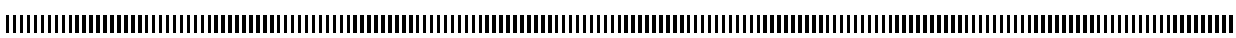
|

CARI'10

\title{
Two-Aircraft Acoustic Optimal Control Problem: SQP algorithms
}

\author{
F.Nahayo ${ }^{1,2}$ - S.Khardi ${ }^{1}$ - J.Ndimubandi² - M.Haddou ${ }^{3}$ - M.Hamadiche ${ }^{4}$ \\ 1 The French Institute of Science and Technology for Transport, Development and Networks- \\ Laboratory of Transports and Environment (Lyon-France), nahayo.fulgence@ gmail.com \\ 2 Mathematics Department, Faculty of Science, University of Burundi \\ 3 Mathematics and Applications, Mathematics-Physics of Orleans, University of Orleans \\ 4 Laboratory of Fluids Mechanics and Acoustics, University Claude Bernard of Lyon 1
}

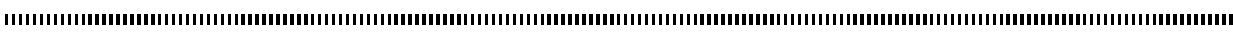

RESUME. Cette contribution vise à développer un modèle mathématique d'optimisation acoustique des trajectoires de vol de deux avions en approche et sans conflit, en minimisant le bruit perçu au sol. Toutes les contraintes de vol des deux avions sont considérées. La dynamique de vol associée au coût génère un problème de contrôle optimal régis par des équations différentielles ordinaires non-linéaires. Pour résoudre ce problème, la théorie des conditions nécessaires d'optimalité pour des problèmes de commande optimale avec contraintes instanées est bien développée. Ceci se caractérise par une solution optimale locale lorsque l'approche newtonienne est utilisée en tenant compte des conditions d'optimalité de Karush-Kuhn-Tucker et la programmation quadratique séquentielle globalisée par région de confiance. Les méthodes SQP sont proposées comme option par KNITRO sous le langage de programmation AMPL. Parmi plusieurs solutions admissibles, il est retenu une trajectoire optimale menant à une réduction du niveau de bruit au sol.

ABSTRACT. This contribution aims to develop an acoustic optimization model of flight paths minimizing two-aircraft perceived noise on the ground. It is about minimizing the noise taking into account all the constraints of flight without conflict. The flight dynamics associated with a cost function generate a non-linear optimal control problem governed by ordinary non-linear differential equations. To solve this problem, the theory of necessary conditions for optimal control problems with instantaneous constraints is well used. This characterizes the optimal solution as a local one when the newtonian approach has been used alongside the optimality conditions of Karush-Kuhn-Tucker and the trust region sequential quadratic programming. The SQP methods are suggested as an option by commercial KNITRO solver under AMPL programming language. Among several possible solution, it was shown that there is an optimal trajectory (for each aircraft) leading to a reduction of noise levels on the ground.

MOTS-CLES : Commande Optimale, Bruit, avions commerciaux, trajectoire, Algorithmes SQP et TRSQP, Programmation non-linéaire.

KEYWORDS : Optimal control problem, Commercial aircraft, noise levels, SQP and TRSQP algorithms, Non-linear programming.

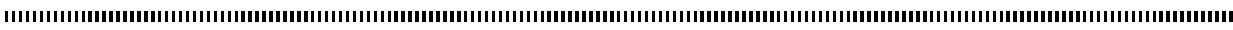

ARIMA, Vol. 14 - pp. 101-123 


\section{Introduction}

Considering the current trend in the field of air transport, economic and environmental considerations related to the rising cost of oil and the need to preserve the environment, impose more severe constraints on the next generation of aircraft [1]. One wants to reach one of the 2020 ACARE objectives [2]. In order to reduce the environmental pollution and noise impact, ACARE requires a 50\% reduction of perceived noise for 2020. This goal represents a difficult scientific and engineering challenge as this requires aerodynamic models and mathematical optimization [3,4]. Some work addressing this problem has been carried out. The majority of this work addresses the problem of minimization of aircraft noise around the airport by considering a single plane $[5,6]$. The other work concerns the stochastic conflict detection for airtraffic management [7], the dynamics of flight [8] and the comprehensive analysis of transport aircraft flight performance [1].

Our aim in this work is the development of a theoretical model of noise optimization while maintening a reliable evolution of the flight procedures of two commercial aircraft on approach. These aircraft are supposed to land successively on the same runway. It is all about the evolution of flight dynamics and minimization of noise for two similar commercial aircraft to the landing taking into account the energy constraint. This model is a non-linear and non-convex optimal control. It is governed by a system of ordinary non-linear differential equations.

For solving this problem, the theory of necessary conditions for optimal control problems with instantaneous constraints on the control and the state is well developped. That characterises the optimal solution as a local solution when the newtonian approach and the sequential quadratic programming are used. The direct optimization methods have proved to be powerful tools for solving optimal control $[9,10]$. The basic idea of direct optimization methods is discretizing the control problem and applying non-linear programming techniques to the resulting finite-dimensional optimization problem. The adopted methods use only control and state variables as optimization variables. The numerical algorithms are usually developped on the basis of first-order necessary optimality conditions. Meanwhile, the second-order sufficient conditions must be checked to ensure the optimality of solutions. Numerically, the second-order sufficient conditions for continuous control problems are very difficult to verify. The alternative solution is to do this for the discretized control problem when using a well-known algebra technique for the optimization problem. It is also important to know the role of the second-order sufficient conditions for sensitivity analysis of the optimal control problem. The control problems are usually subject to disturbances in the data system. Mathematically, the perturbations are described by some parameters in the dynamics, the boundary conditions or in the mixed constraints. The behavior of the optimal solution with the respected perturbations parameters must be analyzed for the stability of the solution.

The new main contribution of this work is the considering of two-arcraft flight dynamics when others autors focuse on one aircraft flight dynamic [5, 6]. One trajectory of a group of two-aircraft is proposed with flight optimal characteristics. Details of the two-aircraft flight dynamic, the noise levels, the constraints, the mathematical model of the two-aircraft acoustic optimal control problem, the sequential quadratic and the trust region sequential quadratic programming method processing are presented in section 2 and 3 while the numerical experiments are presented in the last section. 


\section{Modelization of the two-aircraft optimal control problem}

\subsection{General Formulation}

The minimization of the noise generated by the two planes is an optimal control problem. Let the mathematical general formulation be the following $[11,12]$ :

$$
\left\{\begin{array}{l}
\min _{\mathbf{u} \in \mathbf{U}} J(\mathbf{u}(.), \mathbf{y}(.)) \\
\dot{\mathbf{y}}(t)=\mathbf{f}(\mathbf{u}(t), \mathbf{y}(t)), \mathbf{y}(0)=\mathbf{y}_{0}, \mathbf{u}(0)=\mathbf{u}_{0} \\
\mathbf{k} \mathbf{1}(\mathbf{u}(t), \mathbf{y}(t)) \leq 0 \\
\mathbf{k} \mathbf{2}(\mathbf{u}(t), \mathbf{y}(t)) \geq 0, \forall t \in[0, T]
\end{array}\right.
$$

where $J(\mathbf{u}(),. \mathbf{y}())=.\int_{0}^{T} g(\mathbf{u}(t), \mathbf{y}(t)) d t+\phi(\mathbf{y}(T))$ is the cost function, $\mathbf{y}(t)=\left(\mathbf{y}_{1}(t), \mathbf{y}_{2}(t)\right)^{T}$ is the state of the system, $\mathbf{u}(t)=\left(\mathbf{u}_{1}(t), \mathbf{u}_{2}(t)\right)^{T}$ the control and $J(\mathbf{u}(),. \mathbf{y}($.$) the cost function (noise of the aircraft). \mathbf{k} \mathbf{1}(\mathbf{u}(t), \mathbf{y}(t)) \leq 0, \mathbf{k} \mathbf{2}(\mathbf{u}(t), \mathbf{y}(t)) \geq$ 0 show the mixed constraints. This formulation is general. In the following, we will establish the explicit and realistic form of all the equations cited above.

\subsection{The aircraft dynamic}

By the way, the two aircraft motion equations have been established and here are some considered assumptions [13]. The plane is a solid steel block center of fixed gravity, modeled with a variable mass and its inertia matrix is symmetric. This model presupposes that there is no possible conflict between the two A300 aircraft (The threshold is $5 \mathrm{NM}$ on the horizontal position and $2 \mathrm{e}+3 \mathrm{fts}$ on the vertical one. The landing separation time varies from $45 s$ to $9 \mathrm{e}+1 \mathrm{~s}$ ). The landing law is First Come First Served (FCFS)[14]. The motion of each plane $A_{i}, i:=1,2$ is three dimensional analyzed with 3 frames : the landmark $\left(O, X_{1}, Y_{1}, Z_{1}\right)$, the plane frame $\left(G_{i}, X_{b i}, Y_{b i}, Z_{b i}\right)$ and the aerodynamic one $\left(G_{i}, X_{a i}, Y_{a i}, Z_{a i}\right)$ [7]. The transition between these three frames is shown easily with three successive rotations $[8,15]$. In general, the equations of motion of each aircraft are summarized in two basic relations of mechanics and the fundamental relationship of kinematics :

$$
\begin{aligned}
& \sum \mathbf{F}_{e x t_{i}}-\frac{d m_{i}}{d t} \mathbf{V}_{a i}=\frac{m_{i} d \mathbf{V}_{a i}}{d t} \\
& \sum \mathbf{M}_{e t_{G_{i}}}=J\left(G_{i}, A_{i}\right) \frac{d \mathbf{\Omega}_{i}}{d t}
\end{aligned}
$$

The index $\mathrm{i}=1,2$ reflects the first and second plane. In the system above, $\mathbf{F}_{\text {ext }_{i}}$ represent the external forces acting on the aircraft, $m_{i}(t)$ the mass of the aircraft, $\mathbf{V}_{a i}$ the airspeed of aircraft, $\mathbf{M}_{\text {ext }_{G_{i}}}$ the outside moments of each aircraft, $J\left(G_{i}, A_{i}\right)$ the inertia matrix and $\Omega_{i}$ the angular rotation. The external forces acting on an airplane in flight are : The thrust $\mathbf{F}_{i}=P_{0} \delta_{x i} \frac{\rho}{\rho_{0}}\left(1-M_{i}+\frac{M_{i}^{2}}{2}\right)$, the weight $\mathbf{W}_{i}=m_{i} g \mathbf{Z}_{\mathbf{1}}$, the lift $\mathbf{F}_{p i}=-\frac{1}{2} \rho_{i} S_{i} C_{Z i} V_{a i}^{2} \mathbf{Z}_{a i}$, the $\operatorname{drag} \mathbf{F}_{t i}=-\frac{1}{2} \rho_{i} S_{i} C_{x i} V_{a i}^{2} \mathbf{X}_{a i}$ and the lateral forces $\mathbf{F}_{l i}=\frac{1}{2} \rho_{i} S_{i} C_{y i} V_{a i}^{2} \mathbf{Y}_{a i}$. In these expressions described above, $S_{i}$ is the reference area, 
$C_{x i}$ the coefficient of drag, $V_{a i}$ the speed, $C_{y i}$ the coefficient of lateral force, $C_{z i}$ the coefficient of lift and $\rho_{i}$ the density of air. The time derivation is for an observator attached to frame $R_{O}$ and the equations are written in $R_{a}$. The acceleration is obtained with two time derivations of the position. The relations between the derivatives in the two frames are connected by the well known equation

$$
\left.\frac{d \mathbf{X}}{d t}\right|_{R_{O}}=\left.\frac{d \mathbf{X}}{d t}\right|_{R_{a}}+\boldsymbol{\Omega}_{R_{a} / R_{O}} \times \mathbf{X}
$$

where $\left.\frac{d \mathbf{X}}{d t}\right|_{R_{O}}$ is the derivative with respect to time of the vector $\mathbf{X}$ in the vehicle-carried normal Earth frame $R_{O},\left.\frac{d \mathbf{X}}{d t}\right|_{R_{a}}$ is the derivative with respect to time of the vector $\mathbf{X}$ in the frame $R_{a}, \Omega_{i}$ is the angular velocity of the aircraft and $\boldsymbol{\Omega}_{R_{a} / R_{O}}$ is the angular velocity of the frame $R_{1}$ relative to the frame $R_{0}$. After transformations and simplifications, the system takes the following explicit form :

$$
\left\{\begin{array}{l}
\dot{V_{a_{i}}}=\frac{1}{m_{i}}\left[-m_{i} g \sin \gamma_{a_{i}}-\frac{1}{2} \rho S_{i} V_{a_{i}}^{2} C_{D}+\left(\cos \alpha_{a_{i}}+\sin \alpha_{a_{i}}\right) F_{x_{i}}-\dot{m}_{i} u_{i}\right], \\
\dot{\alpha_{a_{i}}}=\frac{1}{m_{i} V_{a_{i}} \cos \beta_{a_{i}}}\left[m_{i} g \cos \gamma_{a_{i}} \cos \mu_{a_{i}}-\frac{1}{2} \rho S_{i} V_{a_{i}}^{2} C_{L_{i}}+\left[\cos \alpha_{a_{i}}-\sin \alpha_{a_{i}}\right] F_{z_{i}}\right. \\
\left.-\dot{m}_{i} w_{i}\right], \dot{p}_{i}=\frac{C}{A C-E^{2}}\left\{r_{i} q_{i}(B-C)-E p_{i} q_{i}+\frac{1}{2} \rho S_{i} l V_{a_{i}}^{2} C_{l_{i}}\right\} \\
+\frac{E}{A C-E^{2}}\left\{p_{i} q_{i}(A-B)-E r_{i} q_{i}+\frac{1}{2} \rho S_{i} l V_{a_{i}}^{2} C_{n i}\right\} \\
\dot{q}_{i}=\frac{1}{B}\left\{-r_{i} p_{i}(A-C)-E\left(p_{i}^{2}-r_{i}^{2}\right)+\frac{1}{2} \rho S_{i} l V_{a_{i}}^{2} C_{m i}\right\}, \\
\dot{r}_{i}=\frac{E}{A C-E^{2}}\left\{r_{i} q_{i}(B-C)+E p_{i} q_{i}+\frac{1}{2} \rho S_{i} l V_{a_{i}}^{2} C_{l_{i}}+\frac{A}{A C-E^{2}}\left\{p_{i} q_{i}(A-B)\right.\right. \\
\left.-E r_{i} q_{i}+\frac{1}{2} \rho S_{i} l V_{a_{i}}^{2} C_{n i}\right\}, \dot{X}_{G_{i}}^{o}=V_{a_{i}} \cos \gamma_{a_{i}} \cos \chi_{a_{i}}, \dot{Y}_{G_{i}}^{o}=V_{a_{i}} \cos \gamma_{a_{i}} \sin \chi_{a_{i}}, \\
\dot{Z}_{G_{i}}^{o}=-V_{a_{i}} \sin \gamma_{a_{i}}, \dot{\phi}_{i}=p_{i}+q_{i} \sin \phi_{i} \tan \theta_{i}+r_{i} \cos \phi_{i} \tan \theta_{i}, \\
\dot{\theta}_{i}=q_{i} \cos \phi_{i}-r_{i} \sin \phi_{i}, \dot{\psi}_{i}=\frac{\sin \phi_{i}}{\cos \theta_{i}} q_{i}+\frac{\cos \phi_{i}}{\cos \theta_{i}} r_{i}, \\
\dot{m}_{i}=-2.01 \times 10^{-5} \frac{\left(\Phi-\mu-\frac{K}{\eta_{c}}\right) \sqrt{\Theta}}{\sqrt{5 \eta_{n}\left(1+\eta_{t f} \lambda\right)} \sqrt{G+0.2 M_{i}^{2} \frac{\eta_{d} \lambda}{\eta_{t f}} \lambda}-(1-\lambda) M_{i}} F_{i}
\end{array}\right.
$$

where the expressions $A=I_{x x}, B=I_{y y}, C=I_{z z}, E=I_{x z}$ are the inertia moments of the aircraft, 1 is the aircraft reference length, $g$ is the acceleration due to gravity, $C_{D}=$ $C_{D 0}+k C_{L i}^{2}$ is the drag coefficient, $C_{y i}=C_{y \beta} \beta+C_{y p} \frac{p l}{V}+C_{y r} \frac{r l}{V}+C_{Y \delta_{l}} \delta_{l i}+C_{Y \delta_{n}} \delta_{n i}$ is the lateral forces coefficient, $C_{L i}=C_{L \alpha}\left(\alpha_{a}-\alpha_{a 0}\right)+C_{L \delta_{m}} \delta_{m i}+C_{L M} M_{i}+C_{L q} \frac{q_{a}^{b} l}{V}$ is the lift coefficient, $C_{l i}=C_{l \beta} \beta+C_{l p} \frac{p l}{V}+C_{l r} \frac{r l}{V}+C_{l \delta_{l}} \delta_{l i}+C_{l \delta_{n}} \delta_{n i}$ is the rolling moment coefficient, $C_{m i}=C_{m 0}+C_{m \alpha}\left(\alpha-\alpha_{0}\right)+C_{m \delta_{m}} \delta_{m i}$ is the pitching moment coefficient, $C_{n i}=C_{n \beta} \beta+C_{n p} \frac{p l}{V}+C_{n r} \frac{r l}{V}+C_{n \delta_{l}} \delta_{l i}+C_{n \delta_{n}} \delta_{n i}$ is the yawing moment coefficient, $P_{0}$ is the full thrust, $\rho_{0}$ is the atmospheric density at the ground, $F_{i}=\left(F_{x i}, F_{y i}, F_{z i}\right)$ is the propulsive force, $V_{a i}=\left(u_{i}, v_{i}, w_{i}\right)$ is the aerodynamic speed, $\left(X_{i}, Y_{i}, Z_{i}\right)$ are coordinates of the center of gravity of the aircraft i, $\alpha_{a i}$ is the attack angle, $\theta_{i}$ is the inclination angle 
, $\psi_{i}$ is the cup, $\phi_{i}$ is the roll angle, $\left(p_{i}, q_{i}, r_{i}\right)$ are the aircraft velocity relative to the earth and $m_{i}$ the mass. The angles $\gamma_{a_{i}}, \chi_{a_{i}}, \mu_{a_{i}}$ correspond respectively to the aerodynamic climb angle, the aerodynamic azimuth and the aerodynamic bank angle. The mass change is reflected in the aircraft fuel consumption as described by E. Torenbeek [16] where the specific consumption is

$$
C_{S R}=2.01 \times 10^{-5} \frac{\left(\Phi-\mu-\frac{K}{\eta_{c}}\right) \sqrt{\Theta}}{\sqrt{5 \eta_{n}\left(1+\eta_{t f} \lambda\right)} \sqrt{G+0.2 M_{i}^{2} \frac{\eta_{d}}{\eta_{t f}} \lambda}-(1-\lambda) M_{i}}
$$

with the generator function $G$ :

$$
\begin{aligned}
G & =\left(\Phi-\frac{K}{\eta_{c}}\right)\left(1-\frac{1.01}{\frac{\nu-1}{\nu}(K+\mu)\left(1-\frac{K}{\Phi \eta_{c} \eta_{t}}\right)}\right), \\
K & =\mu\left(\epsilon_{c}^{\frac{\nu-1}{\nu}}-1\right), \mu=1+\frac{\nu-1}{2} M_{i}^{2}
\end{aligned}
$$

The Nomenclature of engine performance variables are given by $\mathrm{G}$ the gas generator power function, G0 the gas generator power function (static, sea level), $\mathrm{K}$ the temperature function of compression process, $M_{i}$ the flight Mach number, T4 the turbine Entry total Temperature, $\mathrm{T} 0$ the ambient temperature at sea level, $\mathrm{T}$ the flight temperature, while the nomenclature of engines yields is $\eta_{c}=0.85$ the isentropic compressor efficiency, $\eta_{d}=1-1.3\left(\frac{0.05}{R e^{\frac{1}{5}}}\right)^{2}\left(\frac{0.5}{M_{i}}\right)^{2} \frac{L}{D}$, the isentropic fan intake duct efficiency, $L$ the duct length, $D$ the inlet diameter, Re the Reynolds number at the entrance of the nozzle, $\eta_{f}=0.86-$ $3.13 \times 10^{-2} M_{i}$ the isentropic fan efficiency, $\eta_{i}=\frac{1+\eta_{d} \frac{\gamma-1}{2} M_{i}^{2}}{1+\frac{\gamma-1}{2} M_{i}^{2}}$ the gas Generator intake stagnation pressure ratio, $\eta_{n}=0.97$ the isentropic efficiency of expansion process in nozzle, $\eta_{t}=0.88$ the isentropic turbine efficiency $\eta_{t f}=\eta_{t} \eta_{f}, \epsilon_{c}$ the overall pressure ratio (compressor), $\nu$ the ratio of specific heats $\nu=1.4, \lambda$ the bypass ratio, $\mu$ the ratio of stagnation to static temperature of ambient air, $\Phi$ the nondimensional turbine entry temperature $\Phi=\frac{T_{4}}{T}$ and $\Theta$ the relative ambient temperature $\Theta=\frac{T}{T_{0}}$. For now, let us return to the second equation of (1). Considering the aircraft dynamic, one transforms the system described above according to state $: \frac{d y_{i}(t)}{d t}=f_{i}\left(y_{i}(t), u_{i}(t)\right), i=1,2$ when

$$
\begin{aligned}
& \mathbf{y}_{i}:\left[t_{0}, t_{f}\right] \longrightarrow \mathbf{R}^{\mathbf{1 2}}, \\
& \begin{aligned}
\mathbf{y}_{i}(t)= & \left(\alpha_{a i}(t), \theta_{i}(t), \psi_{i}(t), \phi_{i}(t), V_{a_{i}}(t), X_{G_{i}}(t), Y_{G_{i}}(t), Z_{G_{i}}(t),\right. \\
& \left.p_{i}(t), q_{i}(t), r_{i}(t), m_{i}(t)\right)
\end{aligned}
\end{aligned}
$$

is the state vector where the expressions $\alpha_{a i}(t), \theta_{i}(t), \psi_{i}(t), \phi_{i}(t), V_{a_{i}}(t), X_{G_{i}}(t), Y_{G_{i}}(t)$, $Z_{G_{i}}(t), p_{i}(t), q_{i}(t), r_{i}(t), m_{i}(t)$ are respectively the attack angle, the inclination angle, the cup, the roll angle, the airspeed, the position vectors, the roll velocity of the aircraft relative to the earth, the pitch velocity of the aircraft relative to the earth, the yaw velocity of the aircraft relative to the earth and the aircraft mass. The control vector is

$$
\begin{aligned}
& \mathbf{u}_{i}:\left[t_{0}, t_{f}\right] \longrightarrow \mathbf{R}^{\mathbf{4}}, \\
& \mathbf{u}_{i}(t)=\left(\delta_{l_{i}}(t), \delta_{m_{i}(t)}, \delta_{n_{i}}(t), \delta_{x_{i}}(t)\right)
\end{aligned}
$$


where the expressions $\delta_{l_{i}}(t), \delta_{m_{i}(t)}, \delta_{n_{i}}(t), \delta_{x_{i}}(t)$ are respectively the roll control, the pitch control, the yaw control and the thrust one. The second equation in (1) is then written by :

$$
\dot{\mathbf{y}}(t)=\left(\begin{array}{c}
\dot{\mathbf{y}}_{1}(t) \\
\dot{\mathbf{y}}_{2}(t)
\end{array}\right)=\left(\begin{array}{c}
\mathbf{f}_{1}\left(\mathbf{u}_{1}(t), \mathbf{y}_{1}(t)\right) \\
\mathbf{f}_{2}\left(\mathbf{u}_{2}(t), \mathbf{y}_{2}(t)\right)
\end{array}\right), \forall t \in[0, T], \mathbf{y}_{1}(0)=\mathbf{y}_{10}, \mathbf{y}_{2}(0)=\mathbf{y}_{20}
$$

As the explicit dynamics equation is known, it must be associated with the two aircraft noise as a cost function for the optimal control problem. In the following, the explicit formula of the objective function is shown.

\subsection{The objective function}

The calculation of SEL is described by : SEL $=10 \log \frac{1}{t_{o}} \int_{t_{1}}^{t_{2}} 10^{0.1 L_{A, d t}(t)} d t$ where $t_{o}$ is the time reference taken equal to $1 s$ and $\left[t_{1}, t_{2}\right]$ the noise event interval. If $\left[t_{10}, t_{1 f}\right]$ and $\left[t_{20}, t_{2 f}\right]$ are the respective intervals in which the noise of the first and second plane arises, we have :

$$
\begin{aligned}
{\left[t_{10}, t_{20}\right]: S E L_{1} } & =10 \log \left[\frac{1}{t_{o}} \int_{t_{10}}^{t_{20}} 10^{0.1 L_{A 1, d t}(t)} d t\right] \\
{\left[t_{20}, t_{1 f}\right]: S E L_{12} } & =S E L_{11}+S E L_{21} \\
& =10 \log \left[\frac{1}{t_{o}} \int_{t_{10}}^{t_{1 f}} 10^{0.1 L_{A 1, d t}(t)} d t+\frac{1}{t_{o}} \int_{t_{20}}^{t_{1 f}} 10^{0.1 L_{A 2, d t}(t)} d t\right] \\
{\left[t_{1 f}, t_{2 f}\right]: S E L_{2} } & =10 \log \left[\frac{1}{t_{o}} \int_{t_{20}}^{t_{2 f}} 10^{0.1 L_{A 2, d t}(t)} d t\right] \\
{\left[t_{10}, t_{2 f}\right]: S E L_{G} } & =\frac{\left(t_{20}-t_{10}\right) S E L_{1} \oplus\left(t_{1 f}-t_{20}\right) S E L_{12} \oplus\left(t_{2 f}-t_{1 f}\right) S E L_{2}}{t_{2 f}-t_{10}} \\
& =10 \log \left\{\frac { 1 } { t _ { 2 f } - t _ { 1 0 } } \left[\left(t_{20}-t_{10}\right) \int_{t_{10}}^{t_{20}} 10^{0.1 L_{A 1}(t)} d t\right.\right. \\
& +\left(t_{1 f}-t_{20}\right) \int_{t_{20}}^{t_{1 f}} 10^{0.1 L_{A 1}(t)} d t+\left(t_{1 f}-t_{20}\right) \int_{t_{20}}^{t_{1 f}} 10^{0.1 L_{A 2}(t)} d t \\
& \left.\left.+\left(t_{2 f}-t_{1 f}\right) \int_{t_{1 f}}^{t_{2 f}} 10^{0.1 L_{A 2}(t)} d t\right]\right\}
\end{aligned}
$$

where $S E L_{G}$ is the two-aircraft noise and $\oplus$ means the sound adding. In fact, $L_{A 1}(t)$ is the $A_{1}$ Aircraft jet noise given by the formula [5] : 


$$
\begin{aligned}
L_{A 1}(t) & =141+10 \log \left(\frac{\rho_{1}}{\rho}\right)^{w}+10 \log \left(\frac{V_{e}}{c}\right)^{7.5}+10 \log s_{1}+3 \log \left(\frac{2 s_{1}}{\pi d_{1}^{2}}+0.5\right) \\
& +5 \log \frac{\tau_{1}}{\tau_{2}}+10 \log \left[\left(1-\frac{v_{2}}{v_{1}}\right)^{m e}+1.2 \frac{\left.\left(1+\frac{s_{2} v_{2}^{2}}{s_{1} v_{1}^{2}}\right)^{4}\right]}{\left(1+\frac{s_{2}}{s_{1}}\right)^{3}}\right]-20 \log R+\Delta V \\
& +10 \log \left[\left(\frac{\rho}{\rho_{I S A}}\right)^{2}\left(\frac{c}{c_{I S A}}\right)^{4}\right] ;
\end{aligned}
$$

Where one has : $v_{1}$ is the jet speed at the entrance of the nozzle, $v_{2}$ the speed jet at the nozzle exit, $\tau_{1}$ the inlet temperature of the nozzle, $\tau_{2}$ the temperature at the nozzle exit, $\rho$ the density of air, $\rho_{1}$ the atmospheric density at the entrance of the nozzle, $\rho_{I S A}$ the atmospheric density at ground, $s_{1}$ the fully expanded primary (inner) jet area, $s_{2}$ the fully expanded secondary (outer) area jet, $d_{1}$ the inlet diameter of the nozzle hydraulic engine, $V_{e}=v_{1}\left[1-\left(V / v_{1}\right) \cos \left(\alpha_{p}\right)\right]^{2 / 3}$ the effective speed ( $\alpha_{p}$ is the angle between the axis of the motor and the axis of the aircraft), $R$ the source observer distance, $w$ the exponent variable defined by $: w=\frac{3\left(V_{e} / c\right)^{3.5}}{0.6+\left(V_{e} / c\right)^{3.5}}-1, c$ the sound velocity $(\mathrm{m} / \mathrm{s}), m$ the exhibiting variable depending on the type of aircraft : $m e=1.1 \sqrt{\frac{s_{2}}{s_{1}}} ; \quad \frac{s_{2}}{s_{1}}<29.7$, $m e=6.0 ; \quad \frac{s_{2}}{s_{1}} \geq 29.7$, the term $\Delta V=-15 \log \left(C_{D}\left(M_{c}, \theta\right)\right)-10 \log (1-M \cos \theta)$, means the Doppler convection when $C_{D}\left(M_{c}, \theta\right)=\left[\left(1+M_{c} \cos \theta\right)^{2}+0.04 M_{c}^{2}\right], M$ the Mac Number aircraft $M_{c}$ the convection Mac Number : $M_{c}=0.62\left(v_{1}-V \cos \left(\alpha_{p}\right)\right) / c$, $\theta$ is the Beam angle.

The expression $L_{A 2}(t)$ is the noise of the aircraft $A_{2}$ and it is written as above. By injecting the equation (8) into (7), we have the objective function $J_{G 12}(\mathbf{y}(),. \mathbf{u}())=.S E L_{G}=\int_{t^{\prime}} g(\mathbf{y}(t), \mathbf{u}(t), t) d t . S E L_{G}$ means the two-aircraft noise. The first relation of equation (1) is then written :

$$
\min _{\mathbf{u} \in \mathbf{U}} J_{G 12}(\mathbf{y}(.), \mathbf{u}(.))=\int_{t^{\prime}} g(\mathbf{y}(t), \mathbf{u}(t)) d t+\phi(\mathbf{y}(T))
$$

when the functional $g=S E L_{G}$. If $\phi(\mathbf{y}(T))=0$, the cost function becomes

$$
\begin{aligned}
J_{G 12}(\mathbf{y}(.), \mathbf{u}(.)) & =\int_{t t_{1}}^{t_{1 f}} g_{1}\left(\mathbf{y}_{1}(t), \mathbf{u}_{1}(t), t\right) d t \\
& +\int_{t 20}^{t_{1 f}} g_{12}\left(\mathbf{y}_{1}(t), \mathbf{u}_{1}(t), \mathbf{y}_{2}(t), \mathbf{u}_{2}(t), t\right) d t \\
& +\int_{t 20}^{t_{2 f}} g_{2}\left(\mathbf{y}_{2}(t), \mathbf{u}_{2}(t), t\right) d t
\end{aligned}
$$

The function $g_{12}(t)$ above reflects the coupling between the two-planes noise levels. Returning to the third equation of system (1), one has the constraints. In the following section, the exact formulation of this equation will be shown. 


\subsection{Constraints}

The considered constraints concern aircraft flight speeds and altitudes, flight angles and control positions, energy constraint, aircraft separation, flight velocities of aircraft relative to the earth and the aircraft mass. Some constraints are shared for the two aircraft, others are not.

1) The vertical separation given by $Z_{G_{12}}^{o}=Z_{G_{2}}^{o}-Z_{G_{1}}^{o}$ where $Z_{G_{1}}^{o}, Z_{G_{2}}^{o}$ are respectively the altitude of the first and the second aircraft and $Z_{G_{12}}^{o}$ the altitude separation.

2) The horizontal separation $X_{G_{12}}^{o}=X_{G_{1}}^{o}-X_{G_{2}}^{o}[17,18,19]$ where $X_{G_{1}}^{o}, X_{G_{2}}^{o}$ are horizontal positions of the first and the second aircraft and their separation distance.

3) The aircraft speed $V_{a_{i}}$ must be bounded as follows $1.3 V_{s} \leq V_{a_{i}} \leq V_{i f}$ where $V_{s}$ is the stall speed, $V_{i f}$ is the maximum speed and $V_{i o}=1.3 V_{s}$ the minimum speed of the aircraft $A_{i}[20,16]$. The roll velocity of the aircraft relative to the earth $p_{i} \in\left[p_{i 0}, p_{i f}\right]$, the pitch velocity of the aircraft relative to the earth $q_{i} \in\left[q_{i 0}, q_{i f}\right]$ and the yaw velocity of the aircraft relative to the earth $r_{i} \in\left[r_{i 0}, r_{i f}\right]$ are also considered .

4) On the approach, the ICAO standards and aircraft manufacturers require flight angle evolution as follows : attack angle $\alpha_{a_{i}} \in\left[\alpha_{i o}, \alpha_{i f}\right]$, the inclination angle $\theta_{i} \in$ $\left[\theta_{i 0}, \theta_{i f}\right]$ and the roll angle $\phi_{i} \in\left[\phi_{i o}, \phi_{i f}\right]$.

5) The aircraft control $\mathbf{u}(t)=\left(\delta_{l_{i}}(t), \delta_{m_{i}(t)}, \delta_{n_{i}}(t), \delta_{x_{i}}(t)\right)$ keeps still between the position $\delta_{l i 0}$ and $\delta_{l i f}$ for the roll control, $\delta_{m i 0}$ and $\delta_{m i f}$ for the pitch control, $\delta_{n i 0}$ and $\delta_{\text {nif }}$ for the yaw control and $\delta_{x i 0}$ and $\delta_{x i f}$ for the thrust.

6) The mass $m_{i}$ of the aircraft $A_{i}$ is variable $: m_{i 0}<m_{i}<m_{i f}$. This constraint results in energy consumption of the aircraft $[13,21]$.

On the whole, the constraints come together under the relationship :

$$
\begin{aligned}
& \mathbf{k}_{1 i}: \mathbf{R}^{\mathbf{1 2}} \times \mathbf{R}^{\mathbf{4}} \longrightarrow \mathbf{R}^{\mathbf{1 6}}, \mathbf{k}_{1 i}\left(y_{i}(t), u_{i}(t)\right) \leq 0 \\
& \mathbf{k}_{2 i}: \mathbf{R}^{\mathbf{1 2}} \times \mathbf{R}^{\mathbf{4}} \longrightarrow \mathbf{R}^{\mathbf{1 6}}, \mathbf{k}_{2 i}\left(y_{i}(t), u_{i}(t)\right) \geq 0
\end{aligned}
$$

where

$$
\begin{aligned}
& \mathbf{k}_{1 i}\left(\mathbf{y}_{i}(t), \mathbf{u}_{i}(t)\right)=\left(\alpha_{i}(t)-\alpha_{i f}, \theta_{i}(t)-\theta_{i f}, \psi_{i}(t)-\psi_{i f}, \phi_{i}(t)-\phi_{i f}, V_{a_{i}}(t)-V_{a i f},\right. \\
& X_{G_{i}}^{o}(t)-X_{G i f}^{o}, Y_{G_{i}}^{o}(t)-Y_{G i f}^{o}, Z_{G_{i}}^{o}(t)-Z_{G i f}^{o}, p_{i}(t)-p_{i f}, q_{i}(t)-q_{i f} \\
& \left.r_{i}(t)-r_{i f}, \delta_{l_{i}}(t)-\delta_{l_{i} f}, \delta_{m_{i}}(t)-\delta_{m i f}, \delta_{n_{i}}(t)-\delta_{n i f}, \delta_{x_{i}}(t)-\delta_{x i f}, m_{i}(t)-m_{i f}\right) \\
& \mathbf{k}_{2 i}\left(\mathbf{y}_{i}(t), \mathbf{u}_{i}(t)\right)=\left(\alpha_{i}(t)-\alpha_{i 0}, \theta_{i}(t)-\theta_{i 0}, \psi_{i}(t)-\psi_{i 0}, \phi_{i}(t)-\phi_{i 0}, V_{a_{i}}(t)-V_{a i 0},\right. \\
& X_{G_{i}}^{o}(t)-X_{G i 0}^{o}, Y_{G_{i}}^{o}(t)-Y_{G i 0}^{o}, Z_{G_{i}}^{o}(t)-Z_{G i 0}^{o}, p_{i}(t)-p_{i 0}, q_{i}(t)-q_{i 0} \\
& \left.r_{i}(t)-r_{i 0}, \delta_{l_{i}}(t)-\delta_{l_{i} 0}, \delta_{m_{i}}(t)-\delta_{m i 0}, \delta_{n_{i}}(t)-\delta_{n i 0}, \delta_{x_{i}}(t)-\delta_{x i 0}, m_{i}(t)-m_{i 0}\right) .
\end{aligned}
$$

The digital applications considered for the two-aircraft $[5,8,13,16]$ are confined in Table 1 in appendix. 


\subsection{The explicit formula of the two-aircraft optimal control problem}

In this section, by combining relations (3), (6), (7) and (9), the problem (1) takes the following form

$$
\left\{\begin{array}{l}
\min _{\mathbf{u} \in \mathbf{U}} J_{G 12}(\mathbf{y}(.), \mathbf{u}(.))=\int_{t 10}^{t_{1 f}} g_{1} d t+\int_{t 20}^{t_{1 f}} g_{12} d t+\int_{t 20}^{t_{2 f}} g_{2} d t \\
\dot{\mathbf{y}}(t)=f(\mathbf{u}(t), \mathbf{y}(t)), \mathbf{y}(0)=\mathbf{y}_{0}, \mathbf{u}(0)=\mathbf{u}_{0} \\
\mathbf{k}_{1 i}\left(\mathbf{y}_{i}(t), \mathbf{u}_{i}(t)\right) \leq 0 \\
\mathbf{k}_{2 i}\left(\mathbf{y}_{i}(t), \mathbf{u}_{i}(t)\right) \geq 0, \forall t \in\left[t_{10}, t_{2 f}\right]
\end{array}\right.
$$

where

$$
\begin{aligned}
& g_{1}\left(\mathbf{y}_{1}(t), \mathbf{u}_{1}(t)\right)=\left(t_{2 f}-t_{10}\right)^{-1}\left(t_{20}-t_{10}\right) 10^{0.1 L_{A 1}(t)}, \\
& g_{12}(\mathbf{y}(t), \mathbf{u}(t))=\left(t_{2 f}-t_{10}\right)^{-1}\left(t_{1 f}-t_{20}\right) 10^{0.1\left(L_{A 1}(t)+L_{A 2}(t)\right)} \\
& g_{2}\left(\mathbf{y}_{2}(t), \mathbf{u}_{2}(t)\right)=\left(t_{2 f}-t_{10}\right)^{-1}\left(t_{2 f}-t_{1 f}\right) 10^{0.1 L_{A 1}(t)} .
\end{aligned}
$$

For the two-aircraft optimal control problem as posited in relation (11), several possibilities exist for its resolution. In the literature, we find firstly a theory based on direct methods and non-linear programming, secondly a theory based on indirect methods. In this paper, one tests the first theory based on the newton method approach and SQP methods. The main advantage of Newton's method is its quadratic convergence and as for all other recurring methods, just one starting point is needed to initialize the whole iterative process [22].

\section{SQP methods and KKT-optimality conditions}

\subsection{The optimality conditions for the optimal control problem}

In System (11), one has a problem of optimal control with mixed constraints. By putting $\mathbf{x}=(\mathbf{y}, \mathbf{u})$, the problem can be transformed in the following system :

$$
\left\{\begin{array}{l}
\min J_{G 12}(\mathbf{x}(.)) \\
\dot{\mathbf{y}}=f(\mathbf{x}) \\
n_{j}(\mathbf{x}) \leq 0, j \in \Xi \\
n_{j}(\mathbf{x}) \geq 0, j \in \Gamma
\end{array}\right.
$$

The expressions $\Xi, \Gamma$ are the sets of equality and inequality indices. The Lagrangian of the system (12) is defined by the function $L(\mathbf{x}, \lambda)=J_{G P 12}(\mathbf{x})+\lambda^{T}[b(\dot{\mathbf{y}}, \mathbf{x})+n(\mathbf{x})]$ where the vector $\lambda$ is the Lagrange multiplier and $b(\dot{\mathbf{y}}, \mathbf{x})=\dot{\mathbf{y}}-f(\mathbf{x})=0$.

- An inequality constraint $n_{j}$ is active at point $\tilde{\mathbf{y}}=\left(\mathbf{y}^{*}, \mathbf{u}^{*}, t^{*}\right)$ if $n_{j}\left(\mathbf{y}^{*}, \mathbf{u}^{*}, t^{*}\right)=0$. $\Gamma\left(\mathbf{y}^{*}, \mathbf{u}^{*}, t^{*}\right)=\Gamma^{*}$ is the set of indices $j$ corresponding to active constraints in $\tilde{\mathbf{y}}$,

$$
\begin{aligned}
& \Gamma_{*}^{+}=\left\{j \in \Gamma_{*} \mid\left(\lambda_{\Gamma}^{*}\right)_{j}>0\right\} \\
& \Gamma_{*}^{0}=\left\{j \in \Gamma_{*} \mid\left(\lambda_{\Gamma}^{*}\right)_{j}=0\right\}
\end{aligned}
$$

where the constraints of index $\Gamma_{*}^{+}$are highly active and those of $\Gamma_{*}^{0}$ weakly active.

- An element $\tilde{\mathbf{y}} \in \Gamma^{*}$ verify the condition of qualifying for the constraints $n$ if the gradients of active constraint $\nabla n_{\Xi}(\tilde{\mathbf{y}}), \nabla n_{\Gamma}(\tilde{\mathbf{y}})$ are linearly independent. This means that the Jacobian matrix of active constraints in $\tilde{\mathbf{y}}$ is full.

Vol. 14 - pp. 101-123 
- An element $\tilde{\mathbf{y}} \in \Gamma^{*}$ satisfies the qualification condition of Mangasarian-Fromowitz for constraints $n$ in $\tilde{\mathbf{y}}$ if there exists a direction d such that

$$
\begin{aligned}
& \nabla n_{\Xi}(\tilde{\mathbf{y}})^{T} d=0 \\
& \nabla n_{j}(\tilde{\mathbf{y}})^{T} d<0 \forall j \in \Gamma(\tilde{\mathbf{y}})
\end{aligned}
$$

where the gradient $\left\{\nabla n_{\Xi}(\tilde{\mathbf{y}})\right\}$ are linearly independent.

The Karush-Kuhn-Tucker optimality conditions : Consider that $J_{G 12}, n$ functions of $C^{1}$ class and $\tilde{\mathbf{y}}$ a solution of the problem (14) which satisfies a constraints qualification condition. So,there exists $\lambda^{*}$ such that :

$$
\begin{aligned}
& \nabla_{\mathbf{y}} L\left(\tilde{\mathbf{y}}, \lambda^{*}\right)=0 \\
& n_{\Xi}(\tilde{\mathbf{y}})=0 \\
& n_{\Gamma}(\tilde{\mathbf{y}}) \leq 0 \\
& \lambda_{\Gamma}^{*} \geq 0 \\
& \lambda_{\Gamma}^{*} n_{\Gamma}(\tilde{\mathbf{y}})=0 .
\end{aligned}
$$

The (15) equations are called the conditions of Karush-Kuhn-Tucker(KKT). The first equation reflects the optimality, the second and third the feasability conditions. The other two reflect the additional conditions and Lagrange multipliers corresponding to inactive constraints $n j(\tilde{\mathbf{y}})$ are zero. The couple $\left(\tilde{\mathbf{y}}, \lambda^{*}\right)$ such that the KKT conditions are satisfied is called primal-dual solution of (12). So, $\tilde{\mathbf{y}}$ is called a stationary point.

The necessary optimality conditions of second order [23] : Taking $\tilde{\mathbf{y}}$ a local solution of (13) and satisfying a qualification condition, then there exist multipliers $\left(\lambda^{*}\right)$ such that the KKT conditions are verified. So we have $\nabla_{\mathbf{y y}}^{2} L\left(\tilde{y}, \lambda^{*}\right) d . d>0 \forall h \in \mathbf{C}_{*}$ where $\mathbf{C}_{*}$ is a critical cone.

The sufficient optimality conditions of second order [23] : Suppose that there exits $\left(\lambda^{*}\right)$ which satisfy the KKT conditions and such that $\nabla_{\mathbf{y y}}^{2} L\left(\tilde{\mathbf{y}}, \lambda^{*}\right) d . d>0 \forall h \in \mathbf{C}_{*} \backslash\{0\}$. So $\tilde{\mathbf{y}}$ is a local minimum of $(13)$.

\subsection{SQP Method}

The system (11) results in the following equations :

$$
\left\{\begin{array}{l}
\min J_{G 12}(\mathbf{x}(.)) \\
\dot{\mathbf{y}}=f(\mathbf{x}) \\
n_{j}(\mathbf{x}) \leq 0, j \in \Xi \\
n_{j}(\mathbf{x}) \geq 0, j \in \Gamma
\end{array}\right.
$$

The expressions $\Xi, \Gamma$ are the sets of indices of equality and inequality. An SQP method solves a succession of quadratic problems :

$$
\left\{\begin{array}{l}
\min \nabla J\left(\mathbf{x}_{k}, t_{k}\right) \mathbf{d}_{k}+\frac{1}{2} \mathbf{d}_{k}^{T} H_{k} \mathbf{d}_{k} \\
\nabla^{T} b\left(\dot{\mathbf{y}}_{k}, \mathbf{x}_{k}\right) \mathbf{d}_{k}+b\left(\dot{\mathbf{y}}_{k}, \mathbf{x}_{k}\right)=0 \\
\nabla n_{\Xi}\left(\mathbf{x}_{k}, t_{k}\right) \mathbf{d}_{k}+n_{\Xi}\left(\mathbf{x}_{k}, t_{k}\right) \leq 0 \\
n_{\Gamma}\left(\mathbf{x}_{k}, t_{k}\right) \mathbf{d}_{k}+n_{\Gamma}\left(\mathbf{x}_{k}, t_{k}\right) \geq 0
\end{array}\right.
$$


To solve this problem listed above, choose some subsets of indices $\beta_{k} \in \Gamma, \Xi_{k} \in \Xi$. So, the system becomes only system with equality constraints :

$$
\left\{\begin{array}{l}
\min \nabla J\left(\mathbf{x}_{k}, t_{k}\right) \mathbf{d}_{k}+\frac{1}{2} \mathbf{d}_{k}^{T} H_{k} \mathbf{d}_{k} \\
\nabla^{T} b\left(\dot{\mathbf{y}}_{k}, \mathbf{x}_{k}\right) \mathbf{d}_{k}+\mathbf{b}\left(\dot{\mathbf{y}}_{k}, \mathbf{x}_{k}\right)=0 \\
\nabla n_{\Xi_{k}}\left(\mathbf{x}_{k}, t_{k}\right) \mathbf{d}_{k}+n_{\Xi_{k}}\left(\mathbf{x}_{k}, t_{k}\right)=0, \\
\nabla n_{\beta_{k}}\left(\mathbf{x}_{k}, t_{k}\right) \mathbf{d}_{k}+n_{\beta_{k}}\left(\mathbf{x}_{k}, t_{k}\right)=0 .
\end{array}\right.
$$

The vector $\mathbf{d}_{\mathbf{k}}$ is a primal-dual displacement and $H_{k}$ the Hessian matrix of the Lagrangian. We used a method of Newtonian approach[24].

\subsection{SQP algorithm and added transfomations}

1) Choose the eligible initial conditions for the optimal solution of the problem

2) Problem approximation with a quadratic programming problem with linear constraints at time $t_{k}$.

3) Solve the problem for an eligible descent direction at time $t_{k}$

4) Verify the arrest conditions, if $\left(\mathbf{d}_{k}^{T}=0\right)$, write the solution. Otherwise, proceed to the evaluation of the Hessian matrix, the primal and dual variables, do a linear search to find it.

5) Increment the solution vector at time $t_{k+1}$ and return to Step 2.

This algorithm above must be transformed because the two-Aircraft problem is nonconvex. For improving the robustness and global convergence behavior of this SQP algorithm, it must be added with the trust radius of this form :

$$
\left\|D \Delta \mathbf{x}_{k}\right\|_{p} \leq \Delta, p \in[1, \infty]
$$

where D is uniformly bounded and $\mathbf{d}_{k}=\Delta \mathbf{x}_{k}$. The relations (18) and (19) form a quadratic program when $p=\infty$.

So, the trust-region constraint is restated as $-\Delta e \leq D x \leq \Delta e, e=(1,1,1, \ldots, 1)^{T}$.

If $p=2$, one has the quadratic constraint $\Delta \mathbf{x}_{k}^{T} D^{T} D \Delta \mathbf{x}_{k} \leq \Delta^{2}$. In the following, we develop the convergence theory for any choice of $p$ just to show the equivalence between the $\|.\|_{p}$ and $\|.\|_{2}$. By the combination of some relation of (17) and the relation (19), all the components of the step are controlled by the trust region. The two-aircraft problem takes the following form

$$
\left\{\begin{array}{l}
\min _{\Delta \mathbf{x}_{k}}\left[Q_{G 12}\left(\mathbf{x}_{k}\right)\right]=\nabla^{T} J_{G 12}\left(\mathbf{x}_{k}\right) \Delta \mathbf{x}_{k}+\frac{1}{2}\left(\Delta \mathbf{x}_{k}\right)^{T} H_{k} \Delta \mathbf{x}_{k} \\
\nabla^{T} \mathbf{b}\left(\dot{\mathbf{y}}_{k}, \mathbf{x}_{k}\right) \Delta \mathbf{x}_{k}+b\left(\dot{\mathbf{y}}_{k}, \mathbf{x}_{k}\right)=0 \\
\nabla^{T} n_{\Xi}\left(\mathbf{x}_{k}\right) \Delta \mathbf{x}_{k}+n_{\Xi}\left(\mathbf{x}_{k}\right) \leq 0 \\
\nabla^{T} n_{\Gamma}\left(\mathbf{x}_{k}\right) \Delta \mathbf{x}_{k}+n_{\Gamma}\left(\mathbf{x}_{k}\right) \geq 0 \\
\left\|D \Delta \mathbf{x}_{k}\right\|_{p} \leq \Delta, p \in[1, \infty]
\end{array}\right.
$$

In some situations, all of the components of the step are not controlled by the trust region because of some hypotheses on $\mathrm{D}$. There is an other alternative which allows the practical SQP methods by using the merit function or the penalty function to measure the worth of each point $\mathrm{x}$.

Several approaches like Byrd-Omojokun and Vardi approaches exist to solve the system (12) [25]. It can also be solved with the KNITRO, the SNOPT and other methods 
[26]. In the latter case, we have an ordinary differential system of non-linear and nonconvex equations. The uniqueness of the solution of the quadratic sub-problem is not guaranteed. It therefore combines the algorithm with a merit function for judging the quality of the displacement. The merit function can therefore offer a way to measure all progress of iterations to the optimum while weighing the importance of constraints on the objective function. It is chosen in $l_{2}$ norm particularly the increased Lagrangian $L_{I}$ because of its smooth character. So, in the equation above, one replaces $L$ by $L_{I}$. Thus, this transforms the $\mathrm{SQP}$ algorithm in sequential quadratic programming with trust region globalization 'TRSQP'. Its principle is that each new iteration must decrease the merit function of the problem for an eligible trust radius. Otherwise, we reduce the trust radius $\Delta \mathbf{x}_{K}$ for computing the new displacement. A descent direction is acceptable if its reduction is emotionally positive. The advantages of the method are that the merit function will circumvent the non-convexity of the problem. This approach shows that only one point is sufficient to start the whole iterative process $[24,27,28]$.

Meanwhile, we use an algorithm called feasibility perturbed SQP in which all iterates $\mathbf{x}_{k}$ are feasible and the merit function is the cost function. Let us consider the perturbation $\Delta \tilde{\mathbf{x}}_{k}$ of the step $\Delta \mathbf{x}_{k}$ such that

1) The relation

$$
\mathbf{x}+\Delta \tilde{\mathbf{x}}_{k} \in \mathbf{F}
$$

where $\mathbf{F}$ is the set of feasible points for (12),

2) The asymptotic exactness relation

$$
\left\|\Delta \mathbf{x}-\Delta \tilde{\mathbf{x}}_{k}\right\|_{2} \leq \phi\left(\left\|\Delta \mathbf{x}_{k}\right\|_{2}\right)\left\|\Delta \mathbf{x}_{k}\right\|_{2}
$$

is satisfied where $\phi: \mathbf{R}^{+} \longrightarrow \mathbf{R}^{+}$with $\phi(0)=0$.

These two conditions are used to prove the convergence of the algorithm and the effectiveness of this method. The advantages gained by maintaining feasible iterates for this method are :

- The trust region restriction (19) is added to the SQP problem (18) without concern that it will yield an infeasible subproblem.

- The objective function $J_{G 12}$ is itself used as a merit function in deciding whether to take a step.

- If the algorithm is terminated early, we will be able to use the latest iterate $\mathbf{x}_{k}$ as a feasible suboptimal point, which in many applications is far preferable to an infeasible suboptimum.

\section{The TRSQP algorithm and convergence analysis}

Assume that for a given SQP step $\Delta \mathbf{x}_{k}$ and its perturbation $\Delta \tilde{\mathbf{x}}_{k}$, the ratio to predict decrease is

$$
r_{k}=\frac{J_{G 12}\left(\mathbf{x}_{k}\right)-J_{G 12}\left(\mathbf{x}_{k}+\Delta \tilde{\mathbf{x}}_{k}\right)}{-Q_{G 12}\left(\Delta \tilde{\mathbf{x}}_{k}\right)}
$$

The two-aircraft acoustic optimal control TRSQP algorithm is written as :

1) Let $\mathbf{x}_{0}(k=0)$ a given starting point, $\bar{\Delta} \geq 1$ the trust region upper bound, $\Delta_{0} \in(0, \bar{\Delta})$ an initial radius, $\epsilon \in\left[\epsilon_{0}, \epsilon_{f}\right)$ and $p \in[1, \infty]$ 
2) Calculate $\Delta \mathbf{x}_{k}$ by solving the system

$$
\left\{\begin{array}{l}
\min _{\Delta \mathbf{x}_{k}}\left[Q_{G 12}\left(\mathbf{x}_{k}\right)\right]=\nabla^{T} J_{G 12}\left(\mathbf{x}_{k}\right) \Delta \mathbf{x}_{k}+\frac{1}{2}\left(\Delta \mathbf{x}_{k}\right)^{T} H_{k} \Delta \mathbf{x}_{k} \\
\nabla^{T} b f b\left(\dot{\mathbf{y}}_{k}, \mathbf{x}_{k}\right) \Delta \mathbf{x}_{k}+\mathbf{b}\left(\dot{\mathbf{y}}_{k}, \mathbf{x}_{k}\right)=0 \\
\nabla^{T} n_{\Xi}\left(\mathbf{x}_{k}\right) \Delta \mathbf{x}_{k}+n_{\Xi}\left(\mathbf{x}_{k}\right) \leq 0 \\
\nabla^{T} n_{\Gamma}\left(\mathbf{x}_{k}\right) \Delta \mathbf{x}_{k}+n_{\Gamma}\left(\mathbf{x}_{k}\right) \geq 0 \\
\left\|D \Delta \mathbf{x}_{k}\right\|_{p} \leq \Delta, p \in[1, \infty]
\end{array}\right.
$$

Seek also $\Delta \tilde{\mathbf{x}}_{k}$ by using the system

$$
\begin{aligned}
& \mathbf{x}+\Delta \tilde{\mathbf{x}}_{k} \in \mathbf{F} \\
& \left\|\Delta \mathbf{x}-\Delta \tilde{\mathbf{x}}_{k}\right\|_{2} \leq \phi\left(\left\|\Delta \mathbf{x}_{k}\right\|_{2}\right)\left\|\Delta \mathbf{x}_{k}\right\|_{2}
\end{aligned}
$$

This algorithm consists to find the zero of the derivative of a cost function using Newton's method (the hessian is approximated by BFGS) as the direction of Newton brought down the cost and seek a direction by the trust region method otherwise. The cost function is a local quadratic model in a trust region.

3) If no such for the perturbed counterpart $\Delta \tilde{\mathbf{x}}_{k}$ is found, the following affectations are considered.

$$
\begin{aligned}
& \Delta \mathbf{x}_{k+1} \leftarrow\left(\frac{1}{2}\right)\left\|D_{k} \Delta \mathbf{x}_{k}\right\|_{p} \\
& \mathbf{x}_{k+1} \leftarrow \mathbf{x}_{k} ; D_{k+1} \leftarrow D_{k} ;
\end{aligned}
$$

4) Otherwise, calculate $r_{k}=\frac{J_{G 12}\left(\mathbf{x}_{k}\right)-J_{G 12}\left(\mathbf{x}_{k}+\Delta \tilde{\mathbf{x}}_{k}\right)}{-Q_{G 12}\left(\Delta \tilde{\mathbf{x}}_{k}\right)}$;

if $r_{k} \leq \epsilon_{f}, \Delta_{k+1} \leftarrow\left(\frac{1}{2}\right)\left\|D_{k} \Delta \mathbf{x}_{k}\right\|_{p} ;$

else if $r_{k}>a_{0} \times \epsilon_{0}$ and $\left\|D_{k} \Delta \mathbf{x}_{k}\right\|_{p}=\Delta_{k}$

$\Delta_{k+1} \leftarrow \min \left(2 \Delta_{k}, \Delta\right)$;

else $\Delta_{k+1} \leftarrow \Delta_{k}$;

5) If $r_{k}>\epsilon \mathbf{x}_{k+1} \leftarrow \mathbf{x}_{k}+\Delta \tilde{\mathbf{x}}_{k}$; Choose the new matrix $D_{k+1}$;

else $\mathbf{x}_{k+1} \leftarrow \mathbf{x}_{k} ; D_{k+1} \leftarrow D_{k}$;

6) end.

At each major iteration a positive definite quasi-Newton approximation of the Hessian of the Lagrangian function, $\mathrm{H}$, is calculated using the BFGS method, where $\lambda_{i}, i=1, \ldots, m$, is an estimate of the Lagrange multipliers.

$$
H_{k+1}=H_{k}+\frac{\mathbf{q}_{k} \mathbf{q}_{k}^{T}}{\mathbf{q}_{k}^{T} \mathbf{s}_{k}}-\frac{H_{k}^{T} \mathbf{s}_{k}^{T} \mathbf{s}_{k} H_{k}}{\mathbf{s}_{k}^{T} H_{k} \mathbf{s}_{k}}
$$

where

$$
\begin{aligned}
\mathbf{s}_{k} & =\mathbf{x}_{k+1}-\mathbf{x}_{k}, \\
\mathbf{q}_{k} & =\left(\nabla J_{G 12}\left(\mathbf{x}_{k+1}+\sum_{j=1}^{n} \lambda_{j} . \nabla n\left(\mathbf{x}_{k+1}\right)+b\left(\mathbf{x}_{k+1}\right)\right)\right. \\
& -\left(\nabla J_{G 12}\left(\mathbf{x}_{k}+\sum_{j=1}^{n} \lambda_{j} \cdot \nabla n\left(\mathbf{x}_{k}\right)+b\left(\mathbf{x}_{k}\right)\right)\right.
\end{aligned}
$$

A positive definite Hessian is maintained providing $\mathbf{q}_{k}^{T} \mathbf{s}_{k}$ is positive at each update and that $H$ is initialized with a positive definite matrix. This algorithm is implemented by 
AMPL language programming and the KNITRO solver [29, 30].

Analysis of the algorithm and its convergence. Let us define the set $\mathbf{F}_{\mathbf{0}}$ as follows :

$$
\begin{aligned}
\mathbf{F}_{\mathbf{0}}= & \left\{\mathbf{x} \mid \nabla^{T} \mathbf{b}(\dot{\mathbf{y}}, \mathbf{x}) \Delta \mathbf{x}+\mathbf{b}(\dot{\mathbf{y}}, \mathbf{x})=0, \nabla^{T} n_{\Xi}(\mathbf{x}) \Delta \mathbf{x}+n_{\Xi}(\mathbf{x})=0\right. \\
& \left.\nabla^{T} n_{\Gamma}(\mathbf{x}) \Delta \mathbf{x}+n_{\Gamma}(\mathbf{x}) \geq 0, J_{G 12}(\mathbf{x}) \leq J_{G 12}\left(\mathbf{x}_{0}\right)\right\} \in \mathbf{F}
\end{aligned}
$$

The trust-region bound $\left\|D \Delta \mathbf{x}_{k}\right\|_{p} \leq \Delta, p \in[1, \infty]$ specifies the following assumption.

1) There exists a constant $\beta$ such that for all points $\mathbf{x} \in \mathbf{F}_{\mathbf{0}}$ and all matrix D used in the algorithm, we have for any $\Delta x$ satisfying the following equations

$$
\nabla^{T} b(\dot{\mathbf{y}}, \mathbf{x}) \Delta \mathbf{x}+\mathbf{b}(\dot{\mathbf{y}}, \mathbf{x})=0, \nabla^{T} n_{\Xi}(\mathbf{x}) \Delta \mathbf{x}+n_{\Xi}(\mathbf{x})=0, \nabla^{T} n_{\Gamma}(\mathbf{x}) \Delta \mathbf{x}+n_{\Gamma}(\mathbf{x}) \geq 0
$$

that

$$
\beta^{-1}\|\Delta \mathbf{x}\|_{2} \leq\|D \Delta \mathbf{x}\|_{p} \leq \beta\|\Delta \mathbf{x}\|_{2}
$$

2) The level set $\mathbf{F}_{\mathbf{0}}$ is bounded and the functions $J_{G 12}, b, \eta$ are twice continuously differentiable in an open neighborhood $\mathbf{M}\left(\mathbf{F}_{\mathbf{0}}\right)$ of this set.

Under certain assumptions as shown in [31], this algorithm is well defined.

In this paragraph, one wants to prove that the algorithm has a convergence to stationary point of (13). If we consider that all assumptions hold for each feasible point $\tilde{x}$ for (12), the Mangasarian-Fromowitz are satisfied for constraints. After all, the KKT optimality conditions are specified and that shows that there is at least a local convergence. With other added conditions as shown in [31], the global convergence is held.

\section{Numerical experiments and results}

The following result are obtained with AMPL (A Mathematical Programming Modeling Language) and KNITRO as a solver. Matlab is requested as the graphic visualization programming language.

Figure 1, 2 and 3 are plotted without considering optimization. These show noise levels around the airport inside to explain why our model is very important and which gain is carried when compared with the actual situation. One considers the following zone : $x=-2500: 250: 2500, y=-2500: 250: 2500, h=0$. As the meshing step is $250 \mathrm{~m}$, we have For each meshing point, a vector of $\mathrm{N}$ values on noise level as the discretization shows. For each observation point, one has a vector of $\mathrm{N}$ noise level values as the discretization shows. It is better to take the maximum value among the $\mathrm{N}$ values of noise level matched with the shortest distance between the observer and each plane. On the runway, the touch down position $(\mathrm{m})$ is $(0,0,0)$. The difference between these noise leves on floor for the first aircraft and the second is that when the first plane hit the ground, the second is still at six hundred meters of altitude as shown by the sepation constraints.

The optimal solution is found with the following KNITRO output optimality conditions :

Multistart stopping, found local optimal solution.

MULTISTART : Best locally optimal point is returned. 


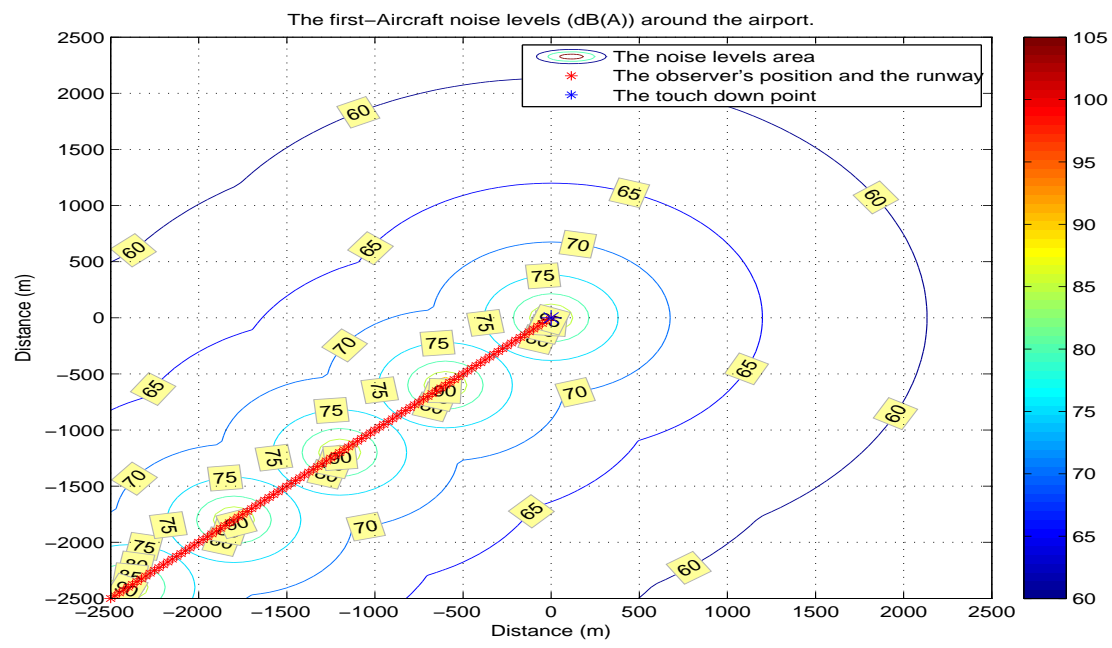

Figure 1. A1 aircraft noise levels

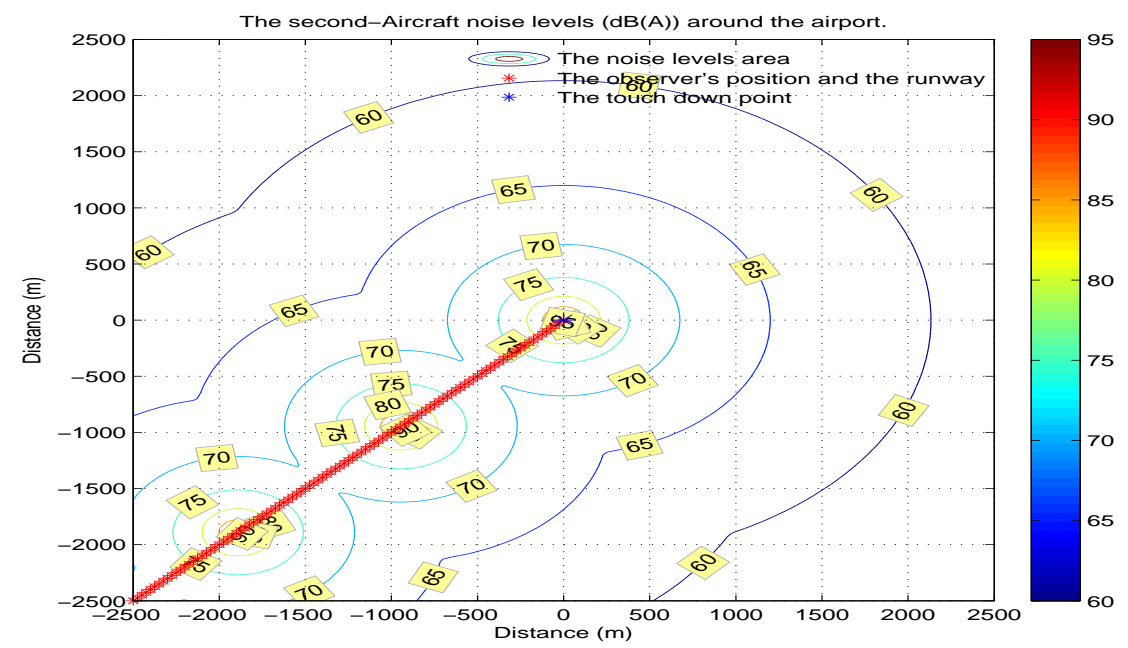

Figure 2. A2 aircraft noise levels

EXIT : Locally optimal solution found.

Final objective value $=5.07801676962590 \mathrm{e}+01$

Final feasibility error $($ abs / rel $)=1.95 \mathrm{e}-07 / 5.11 \mathrm{e}-09$

Final optimality error $(\mathrm{abs} / \mathrm{rel})=6.52 \mathrm{e}-07 / 6.52 \mathrm{e}-07$

Number of iterations $=56$

Number of CG iterations $=114$

Number of function evaluations $=61$ 
116 A R I M A - Volume 14-2011

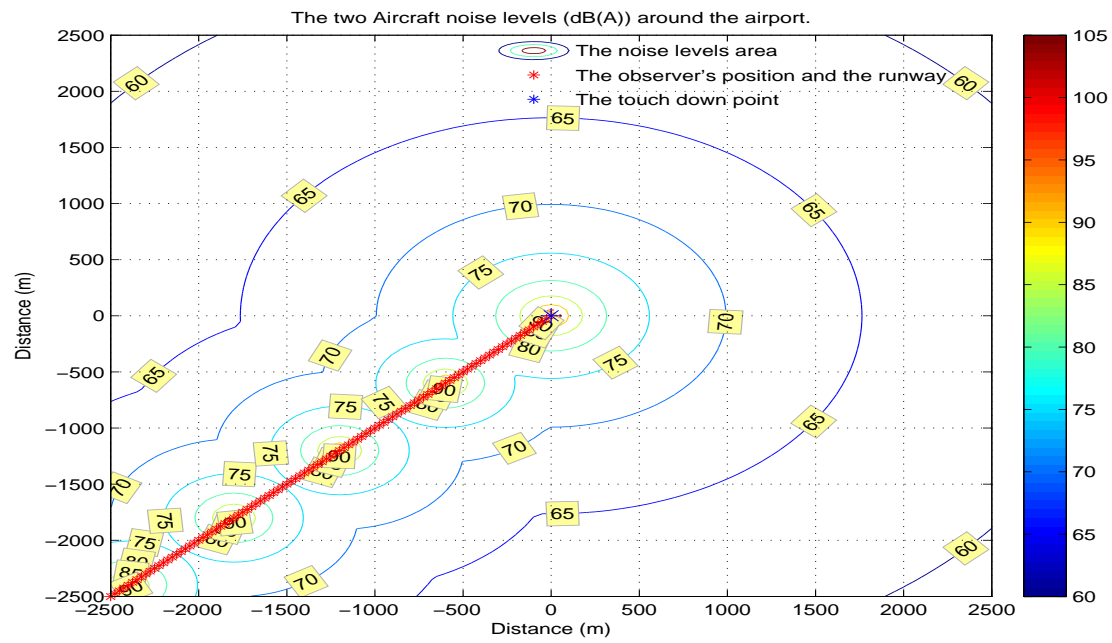

Figure 3. Two-aircraft global noise levels

Numberof gradient evaluations $=57$

Number of Hessian evaluations $=56$

Total program time $(\mathrm{secs})=150.33360(150.289 \mathrm{CPU}$ time $)$

Time spent in evaluations $(\mathrm{secs})=125.97791$

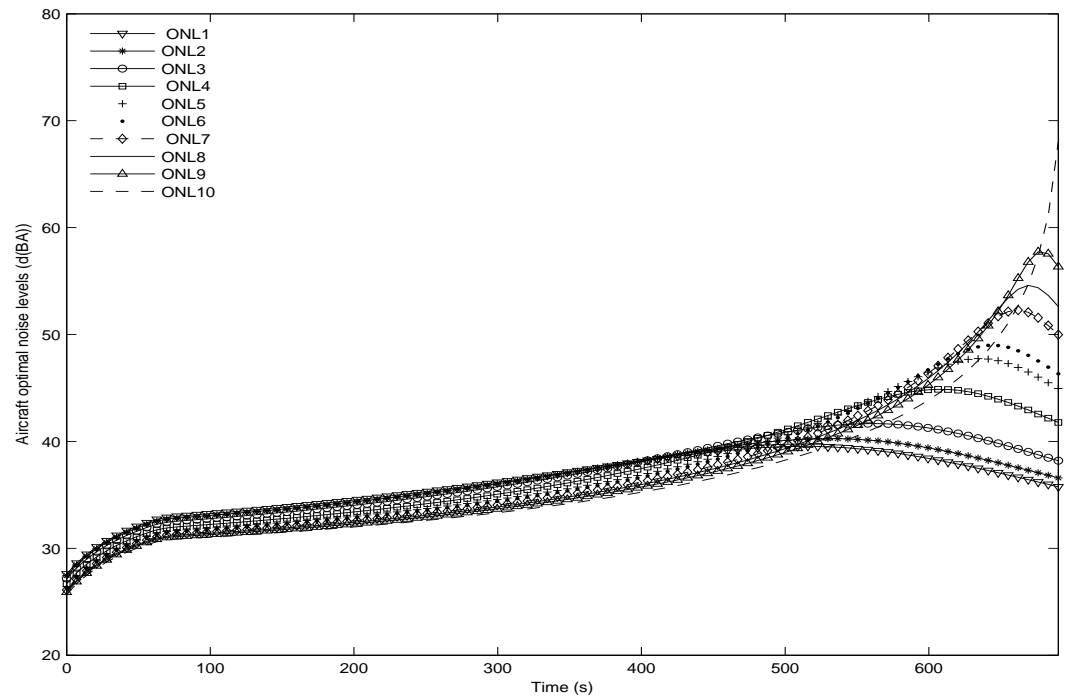

Figure 4. Aircraft optimal noise levels 
Figure 4 shows the noise levels when the optimization is applied and the solutions obtained. The observation positions are $(-20000 \mathrm{~m},-20000 \mathrm{~m}, 0 \mathrm{~m})$ for $O N L_{1}$, $(-19800 m,-19800 m, 0 m)$ for $O N L_{2}, \ldots,(-200 m,-200 m, 0 m)$ for $O N L_{10}$. In this figure, the legend $O N L$ means optimal noise level. As specified, noise level increases and is maximum when the observation point lies below the aircraft. Noise levels decrease gradually as the aircraft moves away from the observation point. This is confirmed by Khardi analysis [32]. By comparison, this result is also close to standard values of jet noise on approach as shown by Harvey [6,33]. To conclude, numerical calculations carried out in this paper are efficient and fitted with experimental and theoretical researches related to acoustical developments.

Figure 5 shows the trajectories which reflect a path in one level flight followed by a continuous descent till the aircraft touch point. The aircrafts' landing procedures are sufficiently separated. It is obvious that each aircraft follows its optimal trajectory when considering the separation distance. Constraints on speeds described in the previous table are considered, allowing a subsequent landing on the same track. Thus, as recommended by ICAO, the security conditions are met and flight procedures are good as shown by the presented results. The maximum altitudes considered are $3500 \mathrm{~m}$ and $4100 \mathrm{~m}$ for the first and the second aircraft. The duration approach is $600 \mathrm{~s}$ for the first aircraft and $690 \mathrm{~s}$ for the second. This figure shows that after some time, we have obtained the same optimal trajectory for the two-aircraft even the procedures are different. This shows the aircraft trajectory resulting from the two trajectories combination. This figure also shows aircraft speed evolution during landing. For the first, the aircraft speed decreases from $200 \mathrm{~m} / \mathrm{s}$ to $140 \mathrm{~m} / \mathrm{s}$ and keeps a constant position till the end of the aircraft landing. This evolution remains the same for the speed of the second aircraft.

Figure 6 shows the aircraft flight angles as recommended by ICAO during aircraft landing. As specified by this figure, the aircraft roll angles oscillate around zero, the flight-path angles are negative and keep the recommended position for aircraft landing procedures. This is the same for the attack angles. Angular variations confirmed the aircraft aerodynamic stability and the flight safety.

Processing calculation provided that the aircraft throttle position is kept constant $(0.6)$ during the landing procedures. The two-aircraft roll velocity $p_{1}, p_{2}$, pitch velocity $q_{1}, q_{2}$ and yaw velocity $r_{1}, r_{2}$, both related to earth frame, are obtained and they have a constant behavior. The behavior of the finesse also confirms the stability of the aircraft flight and reflects the flight procedures characteristic as shown by figures 5 and 6 .

\section{Conclusion}

We have developed a numerical computation of two aircraft optimal control issue. An algorithm for solving the optimal control model has been developed. The algorithm 

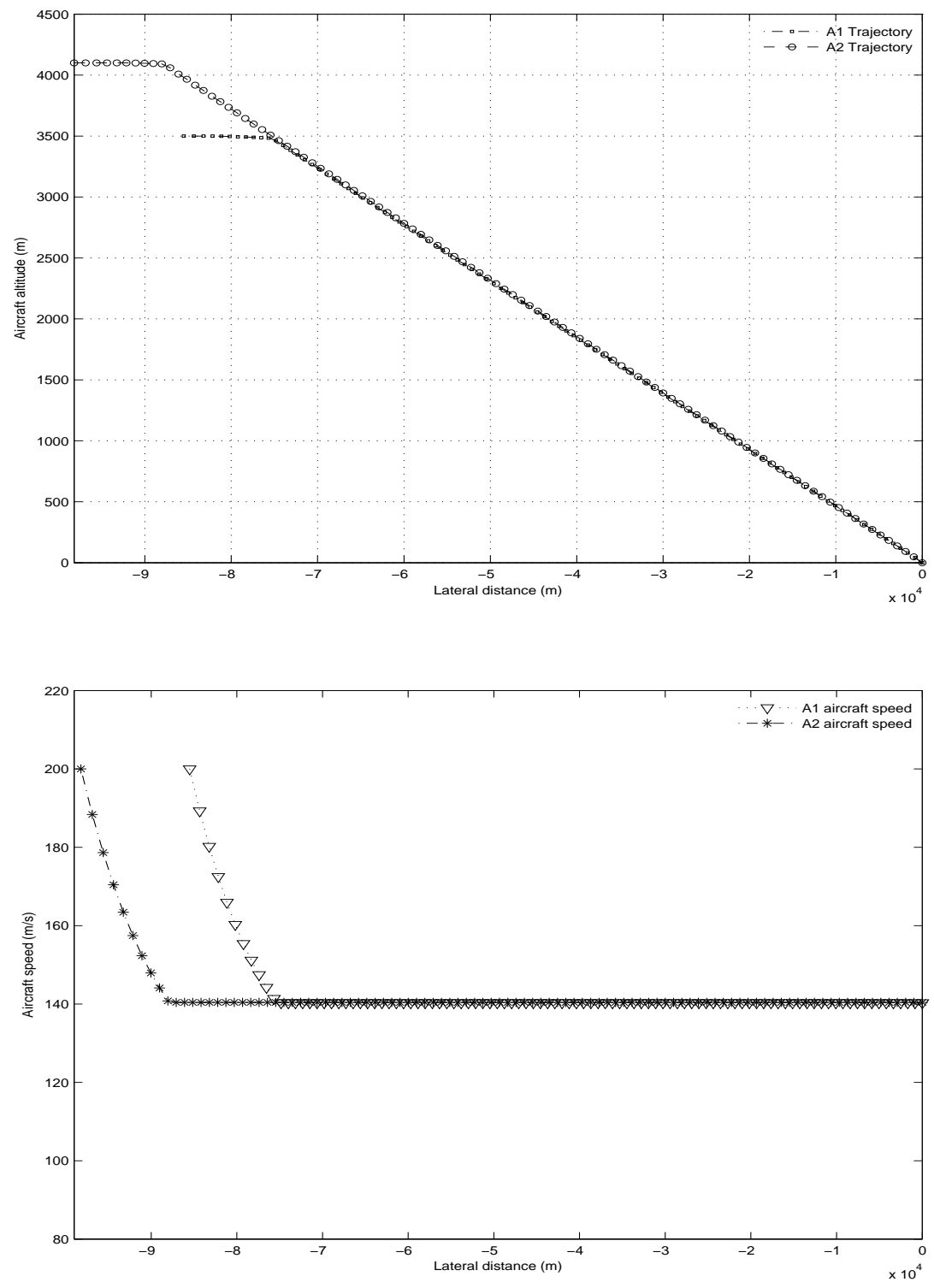

Figure 5. Aircraft optimal flight paths and speeds

minimizes a sequence of merit function using a sub-problem of the quadratic problem at each step for all active constraints to generate a search trust direction for all primal and dual variables. An optimal solution to the discretized problem is found through a local convergence. This solution show a noise reduction during the approach by considering the configuration of several observers. The results obtained present more interesting and acoustically efficient trajectory characteristics and performances. 


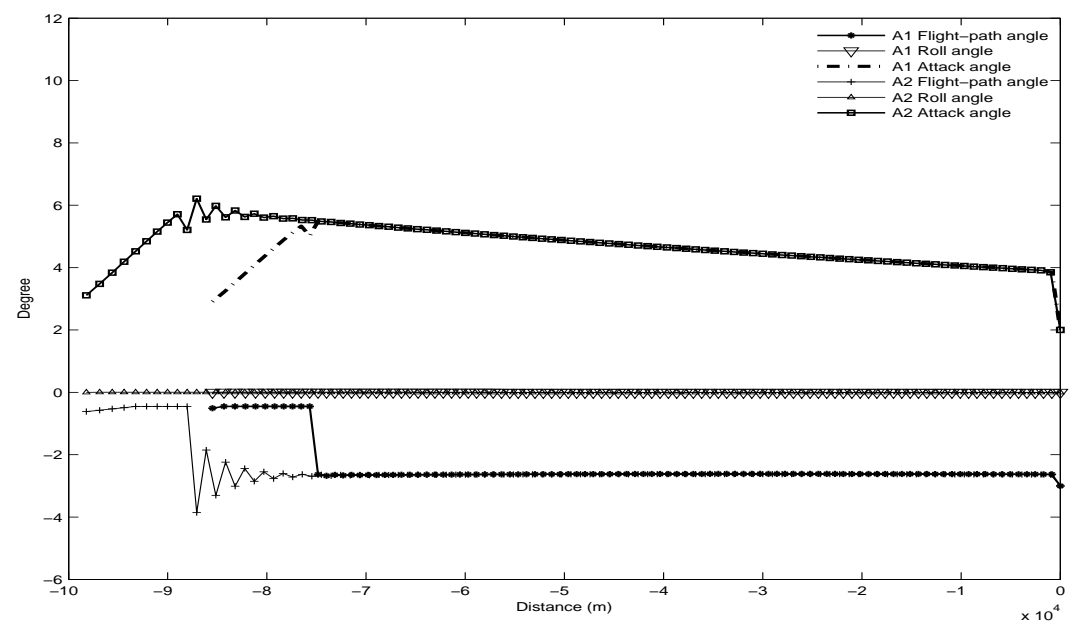

Figure 6. Aircraft flight angles

Further research is needed to generalize the model by combining the perceived noise levels and the fuel consumption by aircraft as objective function using the goal programming technique. This work can also be extended to the case when the second aircraft is delayed $90 \mathrm{~s}$ while the two-aircraft flight paths arise the same.

\section{Références}

[1] A. Filippone. Comprehensive analysis of transport aircraft flight performance. ScinceDirect article, Manchester M60 1QD, vol. 44, pp 192-236, April 2008.

[2] M. Ventre. Les challenges environnementaux pour le transport aérien. SAFRAN, Un leader technologique international, Septembre 2009.

[3] O-I. Zapolozhets and V-I. Tokarev. Predicted Flight Procedures for Minimum Noise Impact. Vol 55, Numéro 2, pp129-149, Elsevier Science Ltd(Great Britain), Ukraine, 1998.

[4] N. Barriety J-P. Jung. La voie de la science, Des outils pour optimiser la conception des avions en phase d'approche. horizon Onera Midi-Pyrenees, Toulouse December 2008.

[5] L. Abdallah. Minimisation des bruits des avions commerciaux sous contraintes physiques et aérodynamique. Thèse de Mathématiques Appliquées de l'UCBL I, Septembre 2007.

[6] L. Abdallah M. Haddou S. Khardi. Optimization of operational aircraft parameters reducing noise emission. Applied Mathematical Sciences, Vol. 4, no 11, 515-535, 2010.

[7] K. Blin. Stochastic conflict detection for air traffic management. Eurocontrol Experimental centre Publications Office, France, April 2000.

Vol. 14 - pp. 101-123 
[8] J-L. Boiffier. The Dynamics of Flight, The Equations. SUPAÉRO(Ecole Nationale Supérieure de l'Aéronautique et de l'Espace) et ONERA-CERT, Toulouse 25 Janvier 1999.

[9] M. Helmut B. Christof. SQP-methods for solving optimal control problems with control and state constraints : adjoint variables, sensitivity analysis and real-time control. Elsivier Science, J. of Computational and Applied Mathematics, Germany, August 2000, vol. 120, pp85-108.

[10] J-B. Rosen A. Barclay, P-E. Gill. SQP methods and their application to numerical optimal control problem, Report NA 97-3. Department of Mathematics, UNiversity of California, San Diego, USA, 1997.

[11] E. Trélat. Commande optimale. University of Orléans, France, 2008.

[12] J-C. Culioli. Introduction à l'optimisation. Ellipses, Ecole Nationale Supérieure des Mines de Paris, 1994.

[13] J-L Boiffier. Dynamique de vol de l'avion. SupAéro, Départements des Aéronefs, Toulouse-Novembre 2001.

[14] C. Stoica. Analyse, représentation et optimisation de la circulation des avions sur une plate-forme aéroportuaire. Thèse de l'Institut national polytechnique de Toulouse-CNRS, Mai, 2005.

[15] R. Ruppli. Algèbre linéaire, idées et méthodes. I.U.T de Dijon, Ellipses, 2002.

[16] E. Roux. Pour une approche analytique de la dynamique du vol. Thèse, SUPAEROONERA, Novembre 2005.

[17] DGAC. Mémento à l'usage des utilisateurs des procédures d'approche et de départ aux instruments. Rapport de la DGAC, 5ềme édition, Août 1995.

[18] H. Sors. Séparation et contrôle aérien. International Virtual Aviation Organization[en ligne]disponible sur http ://academy.ivao.aero/, 15 octobre 2008.

[19] DGAC. Méthodes et minimums de séparations des aéronefs aux procédures. Rapport de la DGAC, Fevrier 2009.

[20] O. Dominique. Cisaillement de vent ou Windshear. http ://www.aviation-fr.info, 2008.

[21] Ifrance. Fiches techniques, historiques et photos d'avions A300-600, A300-600R [en ligne]disponible sur http ://www.ifrance.com. Ifrance.

[22] J. Vignes M. La Porte. Algorithmes numériques, analyse et mise en oeuvre 2, Equations non linéaires. Institut français du pétrole, TECHNIP,[on line] avaible on http ://books.google.com/, Paris, 1980.

[23] M. Bergounioux. Optimisation et Controle des systèmes linéaires, Cours et exercices corrigés. Dunod, 2002.

[24] J-C Gilbert. Eléments d'optimisation différentiable - Théorie et Algorithmes. INRIA Rocquencourt, France, 6 juin 2009.

[25] M. Xavier-Jonsson. Méthodes des points intérieurs et de régions de confience en optimisation non-linéaire et application à la conception des verres ophtamiques progressifs. Thèse de l'Université Paris IV, 2002.

[26] M. Ouriemchi. Résolution de problèmes non-linéaires par les méthods des points intérieurs. Théorie et algorithmes. Thèse de l'Université de Havre, 2005. 
[27] F. Nahayo and S. Khardi. Les méthodes numériques appliquées en optimisation non-linéaire et en commande optimale. Rapport LTE $N^{\circ}$ : 0911, Novembre 2009.

[28] S. Khardi. Mathematical Model for Advanced CDA and Takeoff Procedures Minimizing Aircraft Environmental Impact. International mathematical Forum, 5, no 36, 1747 - 1774, 2010.

[29] R-A. Waltz R-H. Byrd, J. Nocedal. KNITRO : An integrated Package for nonlinear optimization. University of Colorado[en ligne]disponible sur http ://www.ziena.com,http ://www.ampl.com, February, 2006.

[30] T-D. Plantenga R-A. Waltz. KNITRO user's Manual, Version 5.2. University of Colorado[en ligne]disponible sur http ://www.ziena.com, February, 2008.

[31] J. Tenny Mathew J. Stephen Wright. A feasible trust-region sequential quadratic programming algorithm. Optimization technical report, University of Wisconsin, 2002.

[32] S.Khardi. Reduction of commercial aircraft noise emission around airports. A new environmental challenge. Eur. Transp. Res. Rev., (2009) Vol1, pp175-184.

[33] H. Harvey Hubbard. Aeroacoustics of flight vehicles, Theory and Practices. Volume 1 : Noise sources and Volume 2 : Noise Control. NASA Langley Research Center, Hampton, Virginia 1994. 


\section{Appendix}

In this appendix, we present the technical elements, but useful for understanding the paper. We report here the complete equations to describe the two-aircraft dynamics. All the coefficients in these equations are already defined in this paper. The first aircraft dynamics equations are :

$$
f_{1}=\left\{\begin{array}{l}
\dot{V_{a_{1}}}=\frac{1}{m_{1}}\left[-m_{1} g \sin \gamma_{a_{1}}-\frac{1}{2} \rho S_{i} V_{a_{1}}^{2} C_{D}+\left(\cos \alpha_{a_{1}}+\sin \alpha_{a_{1}}\right) F_{x_{1}}-\dot{m}_{1} u_{1}\right], \\
\dot{\alpha_{a_{1}}}=\frac{1}{m_{1} V_{a_{1}} \cos \beta_{a_{1}}}\left[m_{1} g \cos \gamma_{a_{1}} \cos \mu_{a_{1}}-\frac{1}{2} \rho S_{1} V_{a_{1}}^{2} C_{L_{1}}+\left[\cos \alpha_{a_{1}}-\sin \alpha_{a_{1}}\right] F_{z_{1}}\right. \\
\left.-\dot{m}_{1} w_{1}\right], \dot{p}_{1}=\frac{C}{A C-E^{2}}\left\{r_{1} q_{1}(B-C)-E p_{1} q_{1}+\frac{1}{2} \rho S_{1} l V_{a_{1}}^{2} C_{l_{1}}\right\} \\
+\frac{E}{A C-E^{2}}\left\{p_{1} q_{1}(A-B)-E r_{1} q_{1}+\frac{1}{2} \rho S_{1} l V_{a_{1}}^{2} C_{n 1}\right\}, \\
\dot{q}_{1}=\frac{1}{B}\left\{-r_{1} p_{1}(A-C)-E\left(p_{1}^{2}-r_{1}^{2}\right)+\frac{1}{2} \rho S_{1} l V_{a_{1}}^{2} C_{m 1}\right\}, \\
\dot{r}_{1}=\frac{E}{A C-E^{2}}\left\{r_{1} q_{1}(B-C)+E p_{1} q_{1}+\frac{1}{2} \rho S_{1} l V_{a_{1}}^{2} C_{l_{1}}+\frac{A}{A C-E^{2}}\left\{p_{1} q_{1}(A-B)\right.\right. \\
\left.-E r_{1} q_{i}+\frac{1}{2} \rho S_{1} l V_{a_{1}}^{2} C_{n 1}\right\}, \dot{X}_{G_{1}}^{o}=V_{a_{1}} \cos \gamma_{a_{1}} \cos \chi_{a_{1}}, \dot{Y}_{G_{1}}^{o}=V_{a_{1}} \cos \gamma_{a_{1}} \sin \chi_{a_{1}}, \\
\dot{Z}_{G_{1}}^{o}=-V_{a_{1}} \sin \gamma_{a_{1}}, \dot{\phi}_{1}=p_{1}+q_{1} \sin \phi_{1} \tan \theta_{1}+r_{1} \cos \phi_{1} \tan \theta_{1}, \\
\dot{\theta}_{1}=q_{1} \cos \phi_{1}-r_{1} \sin \phi_{1}, \dot{\psi}_{1}=\frac{\sin \phi_{1}}{\cos \theta_{1}} q_{1}+\frac{\cos \phi_{1}}{\cos \theta_{1}} r_{1}, \\
\dot{m}_{1}=-2.01 \times 10^{-5} \frac{\left(\Phi-\mu-\frac{K}{\eta_{c}}\right) \sqrt{\Theta}}{\sqrt{5 \eta_{n}\left(1+\eta_{t f} \lambda\right)} \sqrt{G+0.2 M_{1}^{2} \frac{\eta_{d}}{\eta_{t f}} \lambda}-(1-\lambda) M_{1}} F_{i} .
\end{array}\right.
$$

The second aircraft dynamics equations are :

$$
f_{2}=\left\{\begin{array}{l}
\dot{V_{a_{2}}}=\frac{1}{m_{2}}\left[-m_{2} g \sin \gamma_{a_{2}}-\frac{1}{2} \rho S_{2} V_{a_{2}}^{2} C_{D}+\left(\cos \alpha_{a_{2}}+\sin \alpha_{a_{2}}\right) F_{x_{2}}-\dot{m}_{2} u_{2}\right], \\
\dot{\alpha_{a_{2}}}=\frac{1}{m_{2} V_{a_{2}} \cos \beta_{a_{2}}}\left[m_{2} g \cos \gamma_{a_{2}} \cos \mu_{a_{2}}-\frac{1}{2} \rho S_{i} V_{a_{2}}^{2} C_{L_{2}}+\left[\cos \alpha_{a_{2}}-\sin \alpha_{a_{2}}\right] F_{z_{2}}\right. \\
\left.-\dot{m}_{2} w_{2}\right], \dot{p}_{2}=\frac{C}{A C-E^{2}}\left\{r_{2} q_{2}(B-C)-E p_{2} q_{2}+\frac{1}{2} \rho S_{2} l V_{a_{2}}^{2} C_{l_{2}}\right\} \\
+\frac{E}{A C-E^{2}}\left\{p_{2} q_{2}(A-B)-E r_{2} q_{2}+\frac{1}{2} \rho S_{2} l V_{a_{2}}^{2} C_{n 2}\right\}, \\
\dot{q}_{2}=\frac{1}{B}\left\{-r_{2} p_{2}(A-C)-E\left(p_{2}^{2}-r_{2}^{2}\right)+\frac{1}{2} \rho S_{2} l V_{a_{2}}^{2} C_{m 2}\right\}, \\
\dot{r}_{2}=\frac{E}{A C-E^{2}}\left\{r_{2} q_{2}(B-C)+E p_{2} q_{2}+\frac{1}{2} \rho S_{2} l V_{a_{2}}^{2} C_{l_{2}}+\frac{A}{A C-E^{2}}\left\{p_{2} q_{2}(A-B)\right.\right. \\
\left.-E r_{2} q_{2}+\frac{1}{2} \rho S_{2} l V_{a_{2}}^{2} C_{n 2}\right\}, \dot{X}_{G_{2}}^{o}=V_{a_{2}} \cos \gamma_{a_{2}} \cos \chi_{a_{2}}, \dot{Y}_{G_{2}}^{o}=V_{a_{2}} \cos \gamma_{a_{2}} \sin \chi_{a_{2}}, \\
\dot{Z}_{G_{2}}^{o}=-V_{a_{2}} \sin \gamma_{a_{2}}, \dot{\phi}_{2}=p_{2}+q_{2} \sin \phi_{2} \tan \theta_{2}+r_{2} \cos \phi_{2} \tan \theta_{2}, \\
\dot{\theta}_{2}=q_{2} \cos \phi_{2}-r_{2} \sin \phi_{2}, \dot{\psi}_{2}=\frac{\sin \phi_{2}}{\cos \theta_{2}} q_{2}+\frac{\cos \phi_{2}}{\cos \theta_{2}} r_{2}, \\
\dot{m}_{2}=-2.01 \times 10^{-5} \frac{\left(\Phi-\mu-\frac{K}{\eta_{c}}\right) \sqrt{\Theta}}{\sqrt{5 \eta_{n}\left(1+\eta_{t f} \lambda\right)} \sqrt{G+0.2 M_{2}^{2} \frac{\eta_{d} \lambda}{\eta_{t f}} \lambda}-(1-\lambda) M_{2}} F_{2} .
\end{array}\right.
$$


The limit numerical values for the two-aircraft flight dynamics are confined in the following table :

\begin{tabular}{|l|l|}
\hline Maximum value & Minimum value \\
\hline$V_{a 1 f}=V_{a 2 f}=200 \mathrm{~m} / \mathrm{s}$ & $V_{a 10}=V_{a 20}=73.45 \mathrm{~m} / \mathrm{s}$ \\
$Z_{G 1 f}^{o}=35 \times 10^{2} \mathrm{~m}$ & $Z_{G 10}^{o}=0 \mathrm{~m}$ \\
$Z_{G 2 f}^{o}=41 \times 10^{2} \mathrm{~m}$ & $Z_{G 20}^{o}=0 \mathrm{~m}$ \\
$\delta_{l 1 f}=\delta_{l 2 f}=0.0174$ & $\delta_{l 10}=\delta_{l 20}=-0.0174$ \\
$\delta_{m 1 f}=\delta_{m 2 f}=0.087$ & $\delta_{m 10}=\delta_{m 20}=0$ \\
$\delta_{n 1 f}=\delta_{n 2 f}=0.314$ & $\delta_{n 10}=\delta_{n 20}=-0.035$ \\
$\delta_{x 1 f}=\delta_{x 2 f}=0.6$ & $\delta_{x 10}=\delta_{x 20}=0.2$ \\
$\alpha_{a 1 f}=\alpha_{a 2 f}=12^{\circ}$ & $\alpha_{a 10}=\alpha_{a 20}=2^{\circ}$ \\
$\theta_{a 1 f}=\theta_{a 2 f}=7^{\circ}$ & $\theta_{a 10}=\theta_{a 20}=-7^{\circ}$ \\
$\gamma_{a 1 f}=\gamma_{a 2 f}=0^{\circ}$ & $\gamma_{a 10}=\gamma_{a 20}=-5^{\circ}$ \\
$\mu_{a 1 f}=\mu_{a 2 f}=3^{\circ}$ & $\mu_{a 10}=\mu_{a 20}=-2^{\circ}$ \\
$\chi_{a 1 f}=\chi_{a 2 f}=5^{\circ}$ & $\chi_{a 10}=\chi_{a 20}=-5^{\circ}$ \\
$\phi_{a 1 f}=\phi_{a 2 f}=1^{\circ}$ & $\phi_{a 10}=\phi_{a 20}=-1^{\circ}$ \\
$\psi_{a 1 f}=\psi_{a 2 f}=3^{\circ}$ & $\psi_{a 10}=\psi_{a 20}=-3^{\circ}$ \\
$t_{1 f}=600 \mathrm{~s}, t_{2 f}=645 \mathrm{~s}$ & $t_{10}=0 \mathrm{~s}, t_{20}=45 \mathrm{~s}$ \\
$m_{10} \simeq 1.1 \times 10^{5} \mathrm{~kg}$, & $m_{1 f} \simeq 1.09055 \times 10^{5} \mathrm{~kg}$, \\
$m_{20} \simeq 1.10071 \times 10^{5} \mathrm{~kg}$ & $m_{2 f} \simeq 1.09126 \times 10^{5} \mathrm{~kg}$ \\
$A=5.555 \times 10^{6} \mathrm{~kg} \mathrm{~m} \mathrm{~m}^{2}$ & $B=9.72 \times 10^{6} \mathrm{~kg} \mathrm{m^{2 }}$ \\
$C=14.51 \times 10^{6} \mathrm{~kg} \mathrm{~m} \mathrm{~m}^{2}$ & $E=-3.3 \times 10^{4} \mathrm{~kg} \mathrm{m^{2 }}$ \\
$p_{1 f}=p_{2 f}=1^{\circ} \mathrm{s}^{-1}$ & $p_{10}=p_{20}=-1^{\circ} \mathrm{s}^{-1}$ \\
$q_{1 f}=q_{2 f}=3.6^{\circ} \mathrm{s}^{-1}$ & $q_{10}=q_{20}=3^{\circ} \mathrm{s}^{-1}$ \\
$r_{1 f}=r_{2 f}=12^{\circ} \mathrm{s}^{-1}$ & $r_{10}=r_{20}=-12^{\circ} \mathrm{s}^{-1}$. \\
$Z_{12 f} \geq 2 \times 10^{3} \mathrm{ft} \simeq 6 \times 10^{2} \mathrm{~m}$ & $Z_{120}=2 \times 10^{3} \mathrm{ft} \simeq 6 \times 10^{2} \mathrm{~m}$ \\
$X_{G_{12 f}} \geq 5 \mathrm{~N} M \simeq 9 \times 10^{3} \mathrm{~m}$ & $X_{G_{120}}=5 \mathrm{NM} \simeq 9 \times 10^{3} \mathrm{~m}$ \\
\hline
\end{tabular}

Table1. Limit digital values for the two-aircraft flight dynamic 